\title{
Non-Gaussian perturbations from multi-field inflation
}

\author{
Laura E. Allen, Sujata Gupta and David Wands \\ Institute of Cosmology and Gravitation, University of Portsmouth, Portsmouth PO1 2EG, United Kingdom
}

\begin{abstract}
We show how primordial bispectrum of density perturbations from inflation may be characterised in terms of manifestly gauge-invariant cosmological perturbations at second order. The primordial metric perturbation, $\zeta$, describing the perturbed expansion of uniform-density hypersurfaces on large scales is related to scalar field perturbations on unperturbed (spatially-flat) hypersurfaces at first- and second-order. The bispectrum of the metric perturbation is thus composed of (i) a local contribution due to the second-order gauge-transformation, and (ii) the instrinsic bispectrum of the field perturbations on spatially flat hypersurfaces. We generalise previous results to allow for scale-dependence of the scalar field power spectra and correlations that can develop between fields on super-Hubble scales.
\end{abstract}

PACS numbers: $98.80 . \mathrm{Cq}$

astro-ph/0509719 v2

\section{INTRODUCTION}

Inhomogeneities in the distribution of matter and radiation in the universe today provide valuable information about the dynamical history and physical processes in the very early Universe. In particular temperature anisotropies in the cosmic microwave background (CMB) sky provide detailed information about the primordial density perturbations at the time of last scattering of the $\mathrm{CMB}$ photons. At present the simplest explanation for the origin of these perturbations seems to be that they originated as quantum fluctuations in one or more light fields during a period of inflation in the very early Universe [1, 2]. These vacuum fluctuations on small (sub-Hubble) scales were stretched to large (super-Hubble) scales by the accelerated expansion, where they subsequently evolved as effectively classical perturbations.

Vacuum fluctuations of massless scalar fields in a de Sitter geometry enter a squeezed state on scales larger than the Hubble length where the decaying mode can be neglected. Thus they can be treated as an effectively classical distribution on large scales with Gaussian statistics. For a Gaussian random field the power spectrum is sufficient to describe all the statistical properties of the distribution. But non-linearities in the evolution of the initial fluctuations will lead to a non-Gaussian distribution. Until recently studies of non-Gaussian perturbations from inflation were largely restricted to self-interactions of scalar fields 3 , 4, 5] neglecting non-linear gravity. Gravitational effects can be included in a stochastic approach using local equations to study the evolution of the coarse-grained inflaton field on long wavelengths $[\underline{6}, 7,8,9]$.

Non-Gaussianities inevitably arise at second-order in cosmological perturbation theory but the nature of the nonGaussianity will depend upon the choice of variables [10. Acquaviva et al [1] constructed a gauge-invariant quantity at second-order on large scales, but its physical interpretation was left open and it was only approximately constant during slow-roll inflation 10, 12]. Maldacena 13] gave an analysis of second-order perturbations from slow-roll inflation working in terms of the field perturbation on unperturbed spatial hypersurfaces and relating these to the curvature perturbations on uniform field hypersurfaces during single field inflation. Both these quantities have a clear physical definition and hence are implicitly gauge-invariant. He showed that there is a curvature perturbation, which becomes constant on large scales and whose bispectrum for squeezed triangles $\left(k_{1} \ll k_{2}, k_{3}\right)$ is proportional to the tilt of the scalar power spectrum, and hence small. Maldacena's result has subsequently been verified by a number of authors 14, 15, 16, 17, 18], going beyond slow-roll [14], and extending it to loop corrections [19] and non-minimally coupled fields 15 . For a recent review see Ref. 20].

Only multiple field models of inflation seem likely to generate significant non-Gaussianity in the density perturbation 5, 16, 21, 22, 23, 24, 25, 26]. In particular Lyth and Rodriguez 22] have recently emphasized that the non-Gaussianity of the curvature perturbation may be simply written down in the so-called " $\delta N$-formalism" [27, 28, 29, 30] where one uses the "separate universes" approach 31] to follow the non-linear evolution on super-Hubble scales in terms of locally homogeneous solutions.

In this paper we show how the non-linear evolution of primordial perturbations from inflation may be characterised in terms of manifestly gauge-invariant perturbations at second-order. We adopt the formalism of Malik and Wands [32 who showed that there is a conserved curvature perturbation at second-order for adiabatic density perturbations on long wavelengths. In section II we define the gauge-invariant curvature perturbation at second-order and give the leading order expressions for the power spectrum and bispectrum. In section III we specialise to the case of adiabatic perturbations on large scales from single-field inflation. We relate the conserved curvature perturbation on large scales to the gauge-invariant field perturbations on spatially flat hypersurfaces during slow-roll inflation, 
consistent with the $\delta N$-formalism. In particular we distinguish the purely local form of the bispectrum generated by the second-order gauge transformation from the intrinsic bispectrum of the field perturbations on unperturbed spatial hypersurfaces. We use a simple argument to estimate the bispectrum of field due to large-scale variations in the local Hubble rate during inflation, and hence recover Maldacena's result for the non-linearity of the curvature perturbation. We generalise these results to the multiple-field case in Section IV, allowing for scale dependence of the power spectra and cross-correlations between the fields which may develop on large scales. These results can be simplified by decomposing the field perturbations into an inflaton component (along the instantaneous background trajectory) and orthogonal isocurvature perturbations [33. We conclude in Section V.

\section{GAUGE-INVARIANT PERTURBATIONS}

Discussions of relativistic perturbations have historically been plagued by gauge-dependence of apparently physical quantities such as the density perturbation. For instance, in a spatially homogeneous background where the density evolves in time, the inhomogeneous density perturbation depends on the choice of time coordinate at first-order, and spatial coordinates at second-order. This "gauge-problem" can be avoided by using a physical choice of gauge and building gauge-invariant definitions of the perturbations in that gauge. Thus Bardeen constructed gauge-invariant cosmological perturbations at first-order 34] and Malik and Wands have shown how to construct gauge-invariant cosmological perturbations at second-order [32]. This still leaves a number of different possible gauge-invariant definitions of the density perturbation, for example, corresponding to different physical gauge choices, though the perturbations are nonetheless gauge-invariant.

We will split any scalar $\phi$ into a spatially homogeneous background and first- and second-order inhomogeneities:

$$
\phi(t, x)=\phi_{0}(t)+\delta_{1} \phi(t, x)+\frac{1}{2} \delta_{2} \phi(t, x) .
$$

The split between first and second order inhomogeneities for any given initial perturbation is arbitrary, so we can use this freedom to specify that the first-order perturbations are Gaussian random fields and any non-Gaussianity is described by the second-order perturbation. In particular we will take the Gaussian part of the perturbations to originate from free field fluctuations at early times during inflation, and assume the second-order perturbations are vanishing in this early time limit.

Under a temporal gauge transformation, $\alpha=\alpha_{1}+\left(\alpha_{2} / 2\right)$, a scalar $\phi$ transforms at first- and second-order as

$$
\begin{aligned}
\delta_{1} \phi & \rightarrow \delta_{1} \phi+\dot{\phi}_{0} \alpha_{1} \\
\delta_{2} \phi & \rightarrow \delta_{2} \phi+\dot{\phi}_{0} \alpha_{2}+\partial_{t}\left(\dot{\phi}_{0} \alpha_{1}+2 \delta_{1} \phi\right) \alpha_{1} .
\end{aligned}
$$

We will adopt the method of Malik and Wands 32] to define gauge-invariant cosmological perturbations up to second order by constructing a physical perturbation in a physically defined coordinate system.

There are (at least) two different ways of writing the perturbed line element on any spatial hypersurfaces:

$$
\begin{aligned}
d s^{2} & =a^{2}(t)\left[(1-2 \psi) \delta_{i j} d x^{i} d x^{j}+2 E_{, i j}+2 F_{(i, j)}+h_{i j}\right], \\
& =a^{2}(t)\left[e^{2 \delta N} \delta_{i j} d x^{i} d x^{j}+2 E_{, i j}+2 F_{(i, j)}+h_{i j}\right],
\end{aligned}
$$

where $F_{i}$ is the divergence-free, vector perturbation, and $h_{i j}$ is the transverse and trace-free tensor perturbation. One may refer to $\psi$ as the curvature perturbation and $\delta N$ as the perturbed expansion, though the two are trivially related as $\psi=\left(e^{2 \delta N}-1\right) / 2$. To first and second order we write

$$
\begin{gathered}
\psi=\psi_{1}+\frac{1}{2} \psi_{2}, \\
\delta N=\delta_{1} N+\frac{1}{2} \delta_{2} N,
\end{gathered}
$$

and hence

$$
\begin{aligned}
& \psi_{1}=-\delta_{1} N \\
& \psi_{2}=-\delta_{2} N-2\left(\delta_{1} N\right)^{2} .
\end{aligned}
$$

The curvature perturbation, $\psi$ or $\delta N$, on uniform-density hypersurfaces is non-linearly conserved on large scales (where gradient terms can be neglected) for adiabatic perturbations as a direct consequence of local energy conservation $[29,30,31,32]$, (see also [35, 36]). Thus we will be particularly interested in calculating this perturbation. 
Most authors [13, 29, 30, 37] have worked in terms of $\delta N$ on uniform-density hypersurfaces, which we denote by

$$
\begin{aligned}
\zeta_{1} & \equiv \delta_{1} N-\frac{H}{\dot{\rho}_{0}} \delta_{1} \rho \\
\zeta_{2} & \equiv \delta_{2} N-\frac{H}{\dot{\rho}_{0}} \delta_{2} \rho+\left(\frac{H \delta_{1} \rho}{\dot{\rho}_{0}}\right)\left[2\left(\frac{\delta_{1} \rho}{\dot{\rho}_{0}}-\frac{\delta_{1} N}{H}\right)+\left(\frac{\dot{H}}{H}-\frac{\ddot{\rho}_{0}}{\dot{\rho}_{0}}\right) \frac{\delta_{1} \rho}{\dot{\rho}_{0}}\right] .
\end{aligned}
$$

This definition leaves a residual dependence at second order on the choice of spatial gauge, i.e., the choice of spatial coordinates threading the uniform-density hypersurfaces. This can be eliminated by a physical choice of spatial gauge at first-order [32], such as working with worldlines orthogonal to the uniform-density hypersurfaces. However in what follows we will consider perturbations on large scales where in any case the residual spatial gauge dependence becomes negligible 29].

Malik and Wands [32] gave a gauge-invariant definition of the curvature perturbation at second order on uniformdensity hypersurfaces, $\left.\tilde{\psi}\right|_{\rho}$, and showed this is conserved for adiabatic perturbations on large scales. This is identical to $-\zeta$ defined above at first-order, $\left.\tilde{\psi}_{1}\right|_{\rho}=-\zeta_{1}$, but differs at second order simply due to the difference in choice of curvature or expansion perturbation (9). Thus we have [10]

$$
\left.\tilde{\psi}_{2}\right|_{\rho}=-\left(\zeta_{2}+2 \zeta_{1}^{2}\right)
$$

Hence both $\zeta$ and $\left.\tilde{\psi}\right|_{\rho}$ are conserved at second order for adiabatic perturbations on large scales.

For a stochastic random field, as would be expected to arise from vacuum fluctuatuations during inflation in the early universe, the perturbations are most usefully described in terms of their power spectrum, and higher-order moments, in Fourier space. At linear order the Fourier coefficients of $\zeta$ are defined by the same equation (10) as in real space, while at second order we have

$$
\zeta_{2, \mathbf{k}} \equiv \delta_{2} N_{\mathbf{k}}-\frac{H}{\dot{\rho}_{0}} \delta_{2} \rho_{\mathbf{k}}+\left(\left(\frac{H \delta_{1} \rho}{\dot{\rho}_{0}}\right) *\left[2\left(\frac{\delta_{1} \rho}{\dot{\rho}_{0}}-\frac{\delta_{1} N}{H}\right)+\left(\frac{\dot{H}}{H}-\frac{\ddot{\rho}_{0}}{\dot{\rho}_{0}}\right) \frac{\delta_{1} \rho}{\dot{\rho}_{0}}\right]\right)_{\mathbf{k}},
$$

where "*" represents a convolution

$$
(f * g)(\mathbf{k}) \equiv \int_{-\infty}^{\infty} f\left(\mathbf{k}-\mathbf{k}^{\prime}\right) g\left(\mathbf{k}^{\prime}\right) d^{3} k^{\prime}
$$

where $\not^{3} k$ represents $d^{3} k /(2 \pi)^{3}$. We will be using the corresponding notation $\phi^{3}(\mathbf{k})$ to represent $(2 \pi)^{3} \delta^{3}(\mathbf{k})$.

The power spectrum of the perturbation is given by

$$
P_{\zeta}(\mathbf{k}) \varnothing^{3}\left(\mathbf{k}+\mathbf{k}_{\mathbf{1}}\right) \equiv\left\langle\zeta_{\mathbf{k}} \zeta_{\mathbf{k}_{1}}\right\rangle
$$

For an isotropic distribution the power spectrum is a function solely of $k \equiv|\mathbf{k}|$. Note that the variance per logarithmic interval in $k$-space is given by

$$
\mathcal{P}_{\zeta}(k)=\frac{4 \pi k^{3}}{(2 \pi)^{3}} P_{\zeta}(k) .
$$

The bispectrum of the curvature perturbation is

$$
B_{\zeta}\left(\mathbf{k}_{\mathbf{1}}, \mathbf{k}_{\mathbf{2}}\right) \nexists^{3}\left(\mathbf{k}_{\mathbf{1}}+\mathbf{k}_{\mathbf{2}}+\mathbf{k}_{\mathbf{3}}\right) \equiv\left\langle\zeta_{\mathbf{k}_{\mathbf{1}}} \zeta_{\mathbf{k}_{\mathbf{2}}} \zeta_{\mathbf{k}_{\mathbf{3}}}\right\rangle
$$

which by the requirement of statistical isotropy is non-zero only for a triangle of modes, $\mathbf{k}_{\mathbf{1}}+\mathbf{k}_{\mathbf{2}}+\mathbf{k}_{\mathbf{3}}=0$.

The bispectrum is zero for Gaussian perturbations and hence to leading order we have

$$
\begin{aligned}
P_{\zeta}(k) \phi^{3}\left(\mathbf{k}+\mathbf{k}_{\mathbf{1}}\right)= & \left\langle\zeta_{1, \mathbf{k}} \zeta_{1, \mathbf{k}_{\mathbf{1}}}\right\rangle \\
B_{\zeta}\left(\mathbf{k}_{\mathbf{1}}, \mathbf{k}_{\mathbf{2}}\right) \not^{3}\left(\mathbf{k}_{\mathbf{1}}+\mathbf{k}_{\mathbf{2}}+\mathbf{k}_{\mathbf{3}}\right)= & \frac{1}{2}\left[\left\langle\zeta_{1, \mathbf{k}} \zeta_{1, \mathbf{k}^{\prime}} \zeta_{2, \mathbf{k}^{\prime \prime}}\right\rangle\right. \\
& \left.+\left\langle\zeta_{1, \mathbf{k}} \zeta_{2, \mathbf{k}^{\prime}} \zeta_{1, \mathbf{k}^{\prime \prime}}\right\rangle+\left\langle\zeta_{2, \mathbf{k}} \zeta_{1, \mathbf{k}^{\prime}} \zeta_{1, \mathbf{k}^{\prime \prime}}\right\rangle\right]
\end{aligned}
$$

If the second order part of the curvature perturbation can be written purely in terms of the square of the first order part in real space

$$
\zeta_{2}=-\left(6 f_{N L} / 5\right) \zeta_{1}^{2}
$$


where $f_{N L}$ is a function of background parameters then the bispectrum will be given by

$$
B_{\zeta}\left(k_{1}, k_{2}, k_{3}\right)=-\frac{6}{5} f_{N L}\left(P_{\zeta}\left(k_{1}\right) P_{\zeta}\left(k_{2}\right)+P_{\zeta}\left(k_{1}\right) P_{\zeta}\left(k_{3}\right)+P_{\zeta}\left(k_{2}\right) P_{\zeta}\left(k_{3}\right)\right),
$$

calculated for closed triangles, $\mathbf{k}_{\mathbf{1}}+\mathbf{k}_{\mathbf{2}}+\mathbf{k}_{\mathbf{3}}=0 . f_{N L}$ is then a quantifier of this form of "local" non-Gaussianity and is called the non-linearity parameter.

\section{SINGLE-FIELD INFLATION}

We will now calculate the bispectrum for the scalar field perturbations on large-scales in single-field models of inflation, and hence reproduce the bispectrum for the large-scale curvature perturbation, $\zeta, 13$ ].

\section{A. Curvature perturbations from inflaton perturbations}

During single field inflation, driven by field $\varphi$, density perturbations become adiabatic in the large-scale limit at first- 33] or second-order [12]. An important consequence is that $\zeta$ is conserved and the uniform-density hypersurfaces coincide with uniform-field hypersurfaces on large scales. Thus we have

$$
\begin{aligned}
& \zeta_{1}=\delta_{1} N-\frac{H}{\dot{\varphi}} \delta_{1} \varphi \\
& \zeta_{2}=\delta_{2} N-\frac{H}{\dot{\varphi}} \delta_{2} \varphi+\left(\frac{H \delta_{1} \varphi}{\dot{\varphi}}\right)\left[2\left(\frac{\delta_{1} \varphi}{\dot{\varphi}}-\frac{\delta_{1} N}{H}\right)+\left(\frac{\dot{H}}{H}-\frac{\ddot{\varphi}}{\dot{\varphi}}\right) \frac{\delta_{1} \varphi}{\dot{\varphi}}\right] .
\end{aligned}
$$

These expansion perturbations on uniform-field hypersurfaces are closely related to the field perturbations on uniform-expansion hypersurfaces, which have the gauge-invariant definitions

$$
\begin{aligned}
& \delta_{1} Q \equiv \delta_{1} \varphi-\frac{\dot{\varphi}}{H} \delta_{1} N \\
& \delta_{2} Q \equiv \delta_{2} \varphi-\frac{\dot{\varphi}}{H} \delta_{2} N+\left(\frac{\dot{\varphi} \delta_{1} N}{H}\right)\left[2\left(\frac{\delta_{1} N}{H}-\frac{\delta_{1} \varphi}{\dot{\varphi}}\right)+\left(\frac{\ddot{\varphi}}{\dot{\varphi}}-\frac{\dot{H}}{H}\right)\left(\frac{\delta_{1} N}{H}\right)\right] .
\end{aligned}
$$

At first-order $\delta_{1} Q$ is the usual Mukhanov-Sasaki variable [38, 39] used to quantise the semi-classical linear perturbations during inflation, and $\delta_{2} Q$ is its natural extension to second-order $[13,32$.

We can re-write the metric perturbation $\zeta$ in Eqs. (22) and (23) somewhat more succinctly in terms of these gauge-invariant field perturbations, to give (see also Ref. $[40]$ )

$$
\begin{aligned}
\zeta_{1} & =-\frac{H}{\dot{\varphi}} \delta_{1} Q, \\
\zeta_{2} & =-\frac{H}{\dot{\varphi}} \delta_{2} Q-\left(\frac{\dot{H}}{H^{2}}-\frac{\ddot{\varphi}}{H \dot{\varphi}}\right)\left(\frac{H \delta_{1} Q}{\dot{\varphi}}\right)^{2}-2 \dot{\zeta}_{1} \frac{\delta_{1} \varphi}{\dot{\varphi}},
\end{aligned}
$$

where we have used

$$
\frac{\delta_{1} N}{H}-\frac{\dot{\delta_{1} \varphi}}{\dot{\varphi}}=\frac{\dot{\zeta_{1}}}{H}+\left(\frac{\dot{H}}{H^{2}}-\frac{\ddot{\varphi}}{H \dot{\varphi}}\right) \frac{\delta_{1} \varphi}{\dot{\varphi}} .
$$

Introducing the slow-roll parameters

$$
\begin{aligned}
\epsilon & \equiv-\frac{\dot{H}}{H^{2}} \\
\eta & \equiv-\left(\frac{\dot{H}}{H^{2}}+\frac{\ddot{\varphi}}{H \dot{\varphi}}\right)
\end{aligned}
$$


and using the fact that $\dot{\zeta}_{1}=0$ for adiabatic perturbations on large scales, we finally have

$$
\zeta_{2}=-\frac{H}{\dot{\varphi}} \delta_{2} Q+(2 \epsilon-\eta)\left(\frac{H \delta_{1} Q}{\dot{\varphi}}\right)^{2} .
$$

We can show that the non-linear adiabatic curvature perturbation on large scales during inflation can simply be written as the perturbed expansion history, $N=\int H d t$, due to the gauge-invariant field perturbations 22, 29]

$$
\zeta=\frac{d N}{d \varphi} \delta Q+\frac{1}{2} \frac{d^{2} N}{d \varphi^{2}}(\delta Q)^{2}+\ldots
$$

This is a non-linear extension of the $\delta N$-formalism [27, 28] for calculating perturbations from inflation. Hence to first and second order we have

$$
\begin{aligned}
\zeta_{1} & =\frac{d N}{d \varphi} \delta_{1} Q \\
\zeta_{2} & =\frac{d N}{d \varphi} \delta_{2} Q+\frac{d^{2} N}{d \varphi^{2}} \delta_{1} Q^{2}
\end{aligned}
$$

where

$$
\begin{aligned}
\frac{d N}{d \varphi} & =-\frac{H}{\dot{\varphi}} \\
\frac{d^{2} N}{d \varphi^{2}} & =-\left(\frac{\dot{H}}{H^{2}}-\frac{\ddot{\varphi}}{H \dot{\varphi}}\right)\left(\frac{H}{\dot{\varphi}}\right)^{2}=(2 \epsilon-\eta)\left(\frac{H}{\dot{\varphi}}\right)^{2}
\end{aligned}
$$

\section{B. Non-Gaussianity of field perturbations}

During slow-roll inflation the initial state of the scalar field perturbations can be set by imposing the Minkowski vacuum state on small scales (much smaller than the cosmological Hubble scale, $k / a \gg H$ ) for the scalar field perturbations on spatially-flat hypersurfaces, $\delta N=0$. For any weakly-coupled scalar field this gives an effectively Gaussian distribution of initial field fluctuations. For any light field (with effective mass less than the Hubble scale) living in a de Sitter spacetime, the Hubble damping leads to a squeezed state on scales larger than the Hubble length and hence we can treat the field as a classical random field with

$$
P_{Q}\left(k_{*}\right)=\frac{H^{2}}{2 k_{*}^{3}},
$$

and hence $\mathcal{P}_{\zeta}\left(k_{*}\right)=(H / 2 \pi)^{2}$, where a $*$ subscript denotes Hubble-crossing, $k_{*}=a H$.

We will give a simple extension of the argument due to Maldacena [13, 14] to calculate the amplitude of the secondorder (non-Gaussian) contribution to the scalar field perturbation due to gravitational coupling at Hubble-exit.

It will be convenient to write the first-order field perturbation at Hubble exit as

$$
\delta_{1} Q\left(\mathbf{k}_{*}\right)=\frac{H}{2 \pi} \hat{e}_{\mathbf{k}_{*}} .
$$

where $\hat{e}_{\mathbf{k}}$ is a classical Gaussian random variable with unit variance:

$$
\left\langle\hat{e}_{\mathbf{k}} \hat{e}_{\mathbf{k}^{\prime}}\right\rangle=\frac{\not^{3}\left(\mathbf{k}+\mathbf{k}^{\prime}\right)}{4 \pi k^{3}} .
$$

However at second order there is a local correction to the amplitude of vacuum fluctuations at Hubble exit due to first-order perturbations in the local Hubble rate, $\tilde{H}$. This is determined by the local scalar field value due to longer wavelength modes that have already left the horizon

$$
\tilde{H}=H\left(\varphi_{0}\right)+H^{\prime}\left(\varphi_{0}\right) \int_{0}^{k_{c}} \not^{3} k \delta_{1} Q_{\mathbf{k}} .
$$

where $k_{c}<k_{*}$ is a cut-off wave-number. 
Thus for $k_{*} \gg k_{c}$ we have upto second order

$$
\delta Q\left(\mathbf{k}_{*}\right)=\frac{H}{2 \pi} \hat{e}_{\mathbf{k}}+\int \not^{3} k \frac{H^{\prime} \delta_{1} Q_{k}}{2 \pi} \hat{e}_{\mathbf{k}_{*}-\mathbf{k}} .
$$

and hence we can identify the second order scalar field perturbation at Hubble exit:

$$
\frac{1}{2} \delta_{2} Q_{\mathbf{k} *}=\frac{H^{\prime}}{H}\left(\delta_{1} Q \bullet \delta_{1} Q\right)_{\mathbf{k} *},
$$

where we have taken $\delta_{1} Q_{\mathbf{k}_{*}-\mathbf{k}^{\prime}}=(H / 2 \pi) \hat{e}_{\mathbf{k}_{*}-\mathbf{k}^{\prime}}$ for $k_{*} \gg k^{\prime}$ and a "•" represents a cut-off convolution, that is a convolution-type integral defined by

$$
(f \bullet g)_{\mathbf{k}} \equiv \int_{0}^{k_{c}} d^{3} k^{\prime} f_{\mathbf{k}^{\prime}} g_{\mathbf{k}-\mathbf{k}^{\prime}}
$$

where $k_{c} \ll k$.

This effect gives a non-zero bispectrum (17) for the scalar field perturbations. In the squeezed triangle limit, $k_{2} \simeq k_{3}$ and $k_{1} \ll k_{2}$, the correlation of the field perturbations at the point when the two smaller modes cross the horizon $\left(k_{2}=a H\right)$ gives, to leading order,

$$
\left\langle\delta Q_{\mathbf{k}_{1}} \delta Q_{\mathbf{k}_{\mathbf{2}}} \delta Q_{\mathbf{k}_{3}}\right\rangle=\left\langle\delta_{1} Q_{\mathbf{k}_{1}} \frac{1}{2} \delta_{2} Q_{\mathbf{k}_{\mathbf{2}}} \delta_{1} Q_{\mathbf{k}_{3}}\right\rangle+\left\langle\delta_{1} Q_{\mathbf{k}_{1}} \delta_{1} Q_{\mathbf{k}_{2}} \frac{1}{2} \delta_{2} Q_{\mathbf{k}_{3}}\right\rangle,
$$

Note that the second order part of the perturbation on the larger scale, $\delta_{2} Q_{\mathbf{k}_{1}}$, will be uncorrelated with the modes on smaller scales. Substituting our expression (42) for the second-order field perturbation at horizon crossing into Eq. (44) yields

$$
\begin{aligned}
\left\langle\delta Q_{\mathbf{k}_{1}} \delta Q_{\mathbf{k}_{\mathbf{2}}} \delta Q_{\mathbf{k}_{\mathbf{3}}}\right\rangle & =2 \frac{H^{\prime}}{H}\left\langle\delta_{1} Q_{\mathbf{k}_{1}} \delta_{1} Q_{\mathbf{k}_{\mathbf{2}}}\left(\delta_{1} Q \bullet \delta_{1} Q\right)_{\mathbf{k}_{\mathbf{3}}}\right\rangle, \\
& =-2 \epsilon\left(\frac{H}{\dot{\varphi}}\right) P_{Q}\left(k_{1}\right) P_{Q}\left(k_{2}\right) \not^{3}\left(\mathbf{k}_{\mathbf{1}}+\mathbf{k}_{\mathbf{2}}+\mathbf{k}_{\mathbf{3}}\right)
\end{aligned}
$$

Comparing this with the definition of the bispectrum in Eq. (17) we see that

$$
B_{Q}\left(k, k_{*}\right)=-2 \epsilon\left(\frac{H}{\dot{\varphi}}\right) P_{Q}(k) P_{Q}\left(k_{*}\right) .
$$

Note that this is not strictly of the local form given in Eq. (21) due to the asymmetry between long wavelengths, $k_{1}$, and shorter wavelengths, $k_{2}$ and $k_{3}$. However $P(k) \gg P\left(k_{*}\right)$ for $k \ll k_{*}$ as $\mathcal{P}_{\zeta}(k) \propto k^{3} P_{\zeta}(k)$ generated during slow-roll inflation is nearly scale-invariant, and hence this difference is not significant in the squeezed triangle limit.

One can verify that this coincides, in the limit $k_{1} \ll k_{2}, k_{3}$, with the bispectrum for scalar field perturbations given by Seery and Lidsey in Ref. [16] for the case of a scale-invariant spectrum.

\section{Non-Gaussianity of curvature perturbations}

The power spectrum of the curvature perturbation due to inflaton fluctuations during single field inflation is given by the first-order relation (26). Substituting this into Eq. (18) gives

$$
P_{\zeta}(k)=\left(\frac{H}{\dot{\varphi}}\right)^{2} P_{Q}(k)
$$

The power spectrum of scalar field perturbations at Hubble exit is given by Eq. (37) and thereafter the non-linear curvature perturbation remains constant, on super-Hubble scales, for adiabatic perturbations even after slow-roll inflation ends [29]. Thus for super-Hubble modes with $k \ll a H$ we can write the power spectrum in terms of quantities at Hubble-exit

$$
\mathcal{P}_{\zeta}(k) \equiv \frac{4 \pi k^{3}}{(2 \pi)^{3}} P_{\zeta}(k)=\left(\frac{H^{2}}{2 \pi \dot{\varphi}}\right)_{k=a H}^{2}
$$


Slow-roll gives an almost scale-invariant power spectrum of curvature perturbations on super-Hubble scales, whose tilt is given by

$$
n-1 \equiv \frac{d \ln \mathcal{P}_{\zeta}}{d \ln k}=-6 \epsilon+2 \eta
$$

We can write the bispectrum (17) for the curvature perturbation in terms of a local transformation between secondorder gauge-invariant variables, Eq. (31), and the intrinsic bispectrum of the scalar field perturbations. Substituting Eqs. (26) and (31) into Eq. (19) we obtain

$$
B_{\zeta}\left(\mathbf{k}_{\mathbf{1}}, \mathbf{k}_{\mathbf{2}}\right)=-\left(\frac{H}{\dot{\varphi}}\right)^{3} B_{Q}\left(\mathbf{k}_{\mathbf{1}}, \mathbf{k}_{\mathbf{2}}\right)+(2 \epsilon-\eta)\left(P_{\zeta}\left(k_{1}\right) P_{\zeta}\left(k_{2}\right)+P_{\zeta}\left(k_{2}\right) P_{\zeta}\left(k_{3}\right)+P_{\zeta}\left(k_{3}\right) P_{\zeta}\left(k_{1}\right)\right) .
$$

We see that if the field perturbations on spatially flat hypersurfaces were strictly Gaussian at second order, $B_{Q}=0$, then the second order curvature perturbation $\zeta_{2}$ would be of the "local" form defined in Eq. (20) with $f_{N L}=5(\eta-2 \epsilon) / 6$.

In the squeezed triangle limit, $k \ll k_{*}$, the scalar field bispectrum at Hubble exit is given by (47) and thus

$$
B_{\zeta}\left(k, k_{*}\right)=(6 \epsilon-2 \eta) P_{\zeta}(k) P_{\zeta}\left(k_{*}\right),
$$

where we have used $P_{\zeta}(k) \gg P_{\zeta}\left(k_{*}\right)$ for a nearly scale-invariant spectrum. Thus comparing with Eq. (21) in this squeezed triangle limit, we can identify [13]

$$
f_{N L}=\frac{5}{12}(-6 \epsilon+2 \eta)
$$

As noted by several authors [14, 17] this gives a consistency relation between the primordial non-Gaussianity and the tilt of the power spectrum (50)

$$
f_{N L}=\frac{5}{12}(n-1)
$$

which does not rely on slow-roll during inflation, but does rely on the adiabaticity of the perturbations on super-Hubble scales and thus the existence of a conserved curvature perturbation after Hubble-exit.

\section{MULTI-FIELD INFLATION}

This discussion of of the non-Gaussianty of primordial curvature perturbations from inflation can be readily extended to the multi-field [22] case by generalising Eq. (32)

$$
\zeta=\sum_{i} N_{, i} \delta Q_{i}+\frac{1}{2} \sum_{i, j} N_{, i j} \delta Q_{i} \delta Q_{j}+\ldots
$$

where we identify $\zeta$ with the perturbed expansion of a uniform density hypersurface with respect to an initial uniformexpansion hypersurface during inflation, and we define $N_{, i} \equiv d N / d \varphi_{i}$. Hence to first and second order we have

$$
\begin{aligned}
\zeta_{1} & =\sum_{i} N_{, i} \delta_{1} Q_{i} \\
\zeta_{2} & =\sum_{i} N_{, i} \delta_{2} Q_{i}+\sum_{i, j} N_{, i j} \delta_{1} Q_{i} \delta_{1} Q_{j} .
\end{aligned}
$$

The gauge-invariant definition of field perturbations on uniform-expansion hypersurfaces, $\delta Q$, was given in Eqs. (24) and (25).

It is important to realise in the multi-field case that the primordial curvature perturbation is evaluated on a uniformdensity hypersurface at the some primordial epoch after inflation has ended. Thus the integrated expansion to this uniform-density hypersurface as a function of the initial field local values, $N\left(\varphi_{i}\right)$, must in general be calculated not only during inflation, but throughout the subsequent cosmological history, including reheating. Only in the case of adiabatic perturbations on super-Hubble scales, where the perturbed evolution follows the background trajectory in phase space [31, 33], can we equate $\delta N$ with the perturbed expansion at Hubble-exit, independently of the subsequent expansion history, as was done in Eqs. (32) for the single-field case. Nonetheless, with this caveat we can formally 
write down the primordial perturbation in terms of the integrated expansion $N$ and it's dependence on the different fields.

Thus substituting Eqs. (56) and (57) into the definitions of the power spectrum (18) and bispectrum (19), we obtain

$$
\begin{aligned}
P_{\zeta}(k) & =\sum_{j} N_{, i} N_{, j} C_{i j}(k) \\
B_{\zeta}\left(k_{1}, k_{2}, k_{3}\right) & =\sum_{i, j, m} N_{, i} N_{, j} N_{, m} B_{Q}^{i, j, m}\left(k_{1}, k_{2}, k_{3}\right)+\sum_{i, j, m, n} N_{, i} N_{, j} N_{, m n}\left[C_{i m}\left(k_{1}\right) C_{j n}\left(k_{2}\right)+\text { perms }\right]
\end{aligned}
$$

where we define the multi-field cross-correlation and bispectrum at leading order

$$
\begin{aligned}
\left\langle\delta_{1} Q_{i, \mathbf{k}} \delta_{1} Q_{j, \mathbf{k}^{\prime}}\right\rangle & \equiv C_{i j}(k) \phi^{3}\left(\mathbf{k}+\mathbf{k}^{\prime}\right) \\
\left\langle\delta Q_{i, \mathbf{k}_{\mathbf{1}}} \delta Q_{j, \mathbf{k}_{\mathbf{2}}} \delta Q_{m, \mathbf{k}_{\mathbf{3}}}\right\rangle & \equiv B_{Q}^{i, j, m}\left(k_{1}, k_{2}, k_{3}\right) \not^{3}\left(\mathbf{k}_{\mathbf{1}}+\mathbf{k}_{\mathbf{2}}+\mathbf{k}_{\mathbf{3}}\right) .
\end{aligned}
$$

Equation (59) allows for an arbitrary bispectrum, $B_{Q}^{i, j, m}$, for the field perturbations on spatially flat hypersurfaces and generalises the result of Lyth and Rodriguez 22] to allow for correlations between the fields on super-Hubble scales. In principle it does not rely on slow-roll, only that the subsequent expansion can be expressed as a function solely of the local field values. Nonetheless in the rest of this paper we will restrict our analysis to slow-roll in order to calculate the bispectrum of the field perturbations.

\section{A. Slow-roll field perturbations}

The field perturbations originate as vacuum fluctuations on sub-Hubble scales and hence $\delta Q_{i}$ and $\delta Q_{j}$ are independent random variables at Hubble-exit with

$$
\delta_{1} Q_{i, \mathbf{k}_{*}}=\frac{H}{2 \pi} \hat{e}_{i, \mathbf{k}_{*}}
$$

to leading order in a slow-roll expansion, where

$$
\left\langle\hat{e}_{i, \mathbf{k}} \hat{e}_{j, \mathbf{k}^{\prime}}\right\rangle=\frac{\delta_{i j}}{4 \pi k^{3}} \not^{3}\left(\mathbf{k}+\mathbf{k}^{\prime}\right) .
$$

At Hubble-exit we have $C_{i j}\left(k_{*}\right)=\left(H^{2} / 2 k_{*}^{3}\right) \delta_{i j}$, but coupled evolution of linear perturbations on large scales (proportional to $\partial^{2} V / \partial \varphi_{i} \partial \varphi_{j}$ in slow-roll approximation) can give rise to off-diagonal terms in multi-field inflation [41, 42]. Such off-diagonal terms are small in the slow-roll approximation, but their effect can become significant when integrated over many expansion times for long-wavelengths, $k \ll k_{*}$.

Once again we can estimate the bispectrum of the scalar field perturbations in the squeezed triangle limit $k \ll k_{*}$ at Hubble-exit $k_{*}=a H$, due solely to gravitational coupling in slow-roll inflation. Generalising Eq. (42) to multi-field inflation we obtain

$$
\frac{1}{2} \delta_{2} Q_{i, \mathbf{k} *}=\sum_{j} \frac{H_{, j}}{H}\left(\delta_{1} Q_{j} \bullet \delta_{1} Q_{i}\right)_{\mathbf{k} *},
$$

where $H_{, i} \equiv d H / d \varphi_{i}$. Hence for $k_{1} \ll k_{2} \simeq k_{3} \simeq k_{*}$

$$
\begin{aligned}
\left\langle\delta Q_{i, \mathbf{k}_{\mathbf{1}}} \delta Q_{j, \mathbf{k}_{\mathbf{2}}} \delta Q_{m, \mathbf{k}_{\mathbf{3}}}\right\rangle & =\sum_{n} \frac{H_{, n}}{H}\left(\left\langle\delta_{1} Q_{i, \mathbf{k}_{\mathbf{1}}}\left(\delta_{1} Q_{n} \bullet \delta_{1} Q_{j}\right)_{\mathbf{k}_{\mathbf{2}}} \delta_{1} Q_{m, \mathbf{k}_{\mathbf{3}}}\right\rangle+\left\langle\delta_{1} Q_{i, \mathbf{k}_{\mathbf{1}}} \delta_{1} Q_{j, \mathbf{k}_{\mathbf{2}}}\left(\delta_{1} Q_{n} \bullet \delta_{1} Q_{m}\right)_{\mathbf{k}_{\mathbf{3}}}\right\rangle\right) \\
& =\sum_{n} 2 \frac{H_{, n}}{H} C_{i n}\left(k_{1}\right) C_{j m}\left(k_{2}\right) \phi^{3}\left(\mathbf{k}_{\mathbf{1}}+\mathbf{k}_{\mathbf{2}}+\mathbf{k}_{\mathbf{3}}\right)
\end{aligned}
$$

Comparing (65) with the definition of the multi-field bispectrum in Eq. (61) we see that in the squeezed triangle limit $k \ll k_{*}$ at Hubble-exit

$$
B_{Q}^{i, j, m}\left(k, k_{*}\right)=\sum_{n} 2 \frac{H_{, n}}{H} C_{i n}(k) C_{j m}\left(k_{*}\right) .
$$

As we would expect, the bispectrum for field fluctuations due to gravitational coupling is non-zero only when the Hubble rate at Hubble-exit is dependent upon the field values. 
Following Ref. [33] we can use the unperturbed background solution, $\varphi_{i}(t)$, to define the instantaneous adiabatic or "inflaton" perturbation lying along the background trajectory in multi-field inflation.

$$
d \sigma \equiv \frac{\dot{\varphi}_{i}}{\sqrt{\sum_{j} \dot{\varphi}_{j}^{2}}} d \varphi_{i}
$$

All orthogonal directions in field-space describe instantaneous isocurvature or entropy field perturbations [2, 33] which we denote generically by $\delta \chi$.

In the slow-roll approximation the background trajectory is given by the gradient of the potential, or equivalently the Hubble rate. Thus

$$
\sum_{n} \frac{H_{, n}}{H} \delta_{1} Q_{n}=\frac{H_{, \sigma}}{H} \delta_{1} Q_{\sigma}
$$

Thus the bispectrum (66) is only non-zero for long-wavelength field perturbations $\delta Q_{i}$ which are correlated with the instantaneous inflaton direction, $\sigma$ :

$$
B_{Q}^{i, j, m}\left(k, k_{*}\right)=-2 \epsilon \frac{H}{\dot{\sigma}} C_{i \sigma}(k) C_{j m}\left(k_{*}\right),
$$

analogous to the single-field case (47), where the slow-roll parameter (29) is given by

$$
\epsilon=-\frac{d H}{d \sigma} \frac{\dot{\sigma}}{H^{2}} .
$$

Isocurvature field perturbations that remain decoupled from, and hence uncorrelated with, the inflaton perturbation (i.e., $C_{\chi \sigma}(k)=0$ ), retain a purely Gaussian distribution with $B_{\chi}\left(k, k_{*}\right)=0$ unless we consider non-gravitational interactions, neglected here in our slow-roll approximation.

\section{B. Non-Gaussianity of curvature perturbation in slow-roll}

Finally we can write the bispectrum (59) for the curvature perturbation for multi-field slow-roll inflation in the squeezed triangle limit, $k \ll k_{*}$, as

$$
B_{\zeta}\left(k, k_{*}\right)=2 \sum_{i, j} N_{, i} N_{, j}\left(\epsilon N_{, j} N_{, \sigma} C_{i \sigma}(k)+\sum_{n} N_{, j n} C_{i n}(k)\right) P_{Q_{j}}\left(k_{*}\right) .
$$

One verify that this reduces to Eq. (52) in the single field case where we have $N_{, \sigma}$ and $N_{, \sigma \sigma}$ given by Eqs. (35) and (36). This single-field result for the non-Gaussianity still holds in a multi-field setting so long as the expansion history is independent of all the other fields.

An alternative limit is the case where the expansion history is dominated by an isocurvature field, such that $\left|N_{, \chi}\right| \gg\left|N_{, \sigma}\right|$. This would apply for instance to the curvaton scenario [43]. In this case the primordial curvature perturbation is given by

$$
P_{\zeta}(k)=\left(N_{,}\right)^{2} P_{\chi}(k)
$$

There is no non-Gaussianity due to gravitational coupling, $B_{\chi}=0$, for an isocurvature field that remains decoupled from the from the inflaton, so that $C_{\chi \sigma}=0$. Hence the non-Gaussianity of the primordial curvature perturbation (59) is given by

$$
\begin{aligned}
B_{\zeta}\left(k_{1}, k_{2}, k_{3}\right) & =\left(N_{, \chi}\right)^{2} N_{, \chi \chi}\left[P_{\chi}\left(k_{1}\right) P_{\chi}\left(k_{2}\right)+\text { perms }\right], \\
& =\frac{N_{, \chi \chi}}{\left(N_{, \chi}\right)^{2}}\left[P_{\zeta}\left(k_{1}\right) P_{\zeta}\left(k_{2}\right)+\text { perms }\right] .
\end{aligned}
$$

This is purely of the local form (21) and we can identify [22]

$$
f_{N L}=-\frac{5}{6} \frac{N_{, \chi \chi}}{\left(N_{, \chi}\right)^{2}} .
$$

Even in the general case it can be shown that the contribution of the intrinsic non-Gaussianity of the field perturbations to the bispectrum of the curvature perturbations must be small in the slow-roll approximation [24]. 


\section{CONCLUSIONS}

We have shown how the non-Gaussianity of primordial curvature perturbations is described using gauge-invariant cosmological perturbations at second-order. The split between zeroth, first and second-order parts of inhomogeneous fields is arbitrary, however if we expand about a FRW cosmology, the zeroth-order part of cosmological fields can be taken to be spatially homogeneous. In this paper we have further assumed that the first-order part can be taken to be a Gaussian random field, and the second-order part describes the non-linear evolution of inhomogeneities, and hence the non-Gaussian part of the distribution.

The arbitrariness of the choice of coordinates leaves a residual gauge-freedom. Nonetheless one can construct gauge-invariant quantities, at any order, by specifying an unambiguous, physical quantity [32. In particular we have given a manifestly gauge-invariant definition of $\zeta$, the perturbed expansion on uniform density hypersurfaces on large scales, used by many authors to describe the primordial metric perturbation as it remains constant, at any order, for adiabatic perturbations [29, 30, 35, 36]. This is related to the dimensionless density perturbation on spatially flat hypersurfaces, however the non-linear relation between the two leads to a non-Gaussian metric perturbation for a purely Gaussian density perturbation, and vice versa.

In the case of single-field inflation, the field perturbations become adiabatic in the large-scale limit 12$]$ and the primordial $\zeta$ long after inflation has ended can be equated with the perturbed expansion on uniform-field hypersurfaces at Hubble exit. We have shown how this is equivalent to the non-linear extension of the $\delta N$-formalism 22 , 27, 28, 30] which allows one to calculate the primordial perturbation in terms of $\delta Q$, the field perturbations on spatially flat hypersurfaces, at Hubble exit and the unperturbed FRW solution for the integrated expansion, $N(\varphi)$. This formalism is especially compact for multiple fields where the primordial metric perturbation (say, during primordial nucleosynthesis) may be completely different from that at Hubble exit during inflation. On the other hand one should bear in mind that $N\left(\varphi_{i}\right)$ is a non-linear function of several scalar fields which must be determined by solving the background field equations to determine the integrated expansion up to some fixed primordial density as a function of the field values during inflation. Nonetheless Lyth and Rodriguez [22] have recently shown how powerful this method is for describing the non-Gaussianity of the primordial perturbation due to non-linear evolution on super-Hubble scales.

In single- or multi-field inflation one can give the leading-order expression for the bispectrum of the primordial metric perturbation in terms of the power spectrum and the intrinsic bispectrum of the fields at Hubble-exit during inflation. We have used a simple argument to estimate the intrinsic bispectrum of the field perturbations at Hubble exit due to gravity in the limit of degenerate triangles during slow-roll inflation with single or multiple fields, and hence give an expression for the non-linearity of the primordial density perturbations from slow-roll inflation in this limit. In line with previous results we find that the non-Gaussianity of field perturbations is suppressed during slow-roll, and we have shown that it vanishes at leading order for isocurvature perturbations during inflation. Our final result in Eq. (71) generalises earlier work to allow for non-scale invariance of the scalar field power spectra and correlations between the fields that can develop on super-Hubble scales.

\section{Acknowledgements}

The authors are grateful to Karim Malik for useful comments. This work was supported by a PPARC studentship and PPARC grant PPA/G/S/2000/00115.

[1] A. R. Liddle and D. H. Lyth, "Cosmological inflation and large-scale structure," (CUP, 2000).

[2] B. A. Bassett, S. Tsujikawa and D. Wands, arXiv:astro-ph/0507632

[3] H. M. Hodges, G. R. Blumenthal, L. A. Kofman and J. R. Primack, Nucl. Phys. B 335, 197 (1990).

[4] T. Falk, R. Rangarajan and M. Srednicki, Astrophys. J. 403, L1 (1993) arXiv:astro-ph/9208001.

[5] F. Bernardeau and J. P. Uzan, Phys. Rev. D 66, 103506 (2002) arXiv:hep-ph/0207295 ; Phys. Rev. D 67, 121301 (2003) arXiv:astro-ph/0209330.

[6] A. Gangui, F. Lucchin, S. Matarrese and S. Mollerach, Astrophys. J. 430, 447 (1994) arXiv:astro-ph/9312033.

[7] S. Gupta, A. Berera, A. F. Heavens and S. Matarrese, Phys. Rev. D 66, 043510 (2002) arXiv:astro-ph/0205152.

[8] T. Hattori and K. Yamamoto, JCAP 0507, 005 (2005) arXiv:astro-ph/0506373.

[9] G. I. Rigopoulos and E. P. S. Shellard, arXiv:astro-ph/0405185

[10] D. H. Lyth and Y. Rodriguez, Phys. Rev. D 71 (2005) 123508 arXiv:astro-ph/0502578.

[11] V. Acquaviva, N. Bartolo, S. Matarrese and A. Riotto, Nucl. Phys. B 667, 119 (2003) arXiv:astro-ph/0209156.

[12] F. Vernizzi, Phys. Rev. D 71, 061301 (2005) arXiv:astro-ph/0411463. 
[13] J. Maldacena, JHEP 0305 (2003) 013 arXiv:astro-ph/0210603.

[14] P. Creminelli and M. Zaldarriaga, JCAP 0410, 006 (2004) arXiv:astro-ph/0407059.

[15] D. Seery and J. E. Lidsey, JCAP 0506, 003 (2005) arXiv:astro-ph/0503692.

[16] D. Seery and J. E. Lidsey, arXiv:astro-ph/0506056

[17] A. Gruzinov, Phys. Rev. D 71, 027301 (2005) arXiv:astro-ph/0406129.

[18] G. I. Rigopoulos, E. P. S. Shellard and B. W. van Tent, arXiv:astro-ph/0410486

[19] S. Weinberg, Phys. Rev. D 72, 043514 (2005).

[20] N. Bartolo, E. Komatsu, S. Matarrese and A. Riotto, Phys. Rept. 402, 103 (2004) arXiv:astro-ph/0406398.

[21] D. H. Lyth, C. Ungarelli and D. Wands, Phys. Rev. D 67, 023503 (2003) arXiv:astro-ph/0208055.

[22] D. H. Lyth and Y. Rodriguez, arXiv:astro-ph/0504045

[23] G. I. Rigopoulos, E. P. S. Shellard and B. W. van Tent, arXiv:astro-ph/0504508 arXiv:astro-ph/0506704

[24] D. H. Lyth and I. Zaballa, arXiv:astro-ph/0507608

[25] A. D. Linde and V. Mukhanov, Phys. Rev. D 56, 535 (1997) arXiv:astro-ph/9610219.

[26] M. Zaldarriaga, Phys. Rev. D 69, 043508 (2004) arXiv:astro-ph/0306006.

[27] A. A. Starobinsky, Phys. Lett. B 117, 175 (1982).

[28] M. Sasaki and E. D. Stewart, Prog. Theor. Phys. 95, 71 (1996) arXiv:astro-ph/9507001.

[29] D. H. Lyth and D. Wands, Phys. Rev. D 68 (2003) 103515 arXiv:astro-ph/0306498.

[30] D. H. Lyth, K. A. Malik and M. Sasaki, JCAP 0505, 004 (2005) arXiv:astro-ph/0411220.

[31] D. Wands, K. A. Malik, D. H. Lyth and A. R. Liddle, Phys. Rev. D 62 (2000) 043527 arXiv:astro-ph/0003278.

[32] K. A. Malik and D. Wands, Class. Quant. Grav. 21 (2004) L65 arXiv:astro-ph/0307055 .

[33] C. Gordon, D. Wands, B. A. Bassett and R. Maartens, Phys. Rev. D 63, 023506 (2001) arXiv:astro-ph/0009131.

[34] J. M. Bardeen, Phys. Rev. D 22, 1882 (1980).

[35] G. I. Rigopoulos and E. P. S. Shellard, Phys. Rev. D 68, 123518 (2003) arXiv:astro-ph/0306620.

[36] D. Langlois and F. Vernizzi, Phys. Rev. Lett. 95, 091303 (2005) arXiv:astro-ph/0503416; arXiv:astro-ph/0509078

[37] M. Zaldarriaga, Phys. Rev. D 69, 043508 (2004) arXiv:astro-ph/0306006.

[38] V. F. Mukhanov, Sov. Phys. JETP 67, 1297 (1988) [Zh. Eksp. Teor. Fiz. 94N7, 1 (1988)].

[39] M. Sasaki, Prog. Theor. Phys. 76, 1036 (1986).

[40] K. A. Malik, arXiv:astro-ph/0506532

[41] D. Wands, N. Bartolo, S. Matarrese and A. Riotto, Phys. Rev. D 66, 043520 (2002) arXiv:astro-ph/0205253.

[42] N. Bartolo, S. Matarrese and A. Riotto, Phys. Rev. D 64, 083514 (2001) arXiv:astro-ph/0106022.

[43] K. Enqvist and M. S. Sloth, Nucl. Phys. B 626, 395 (2002) arXiv:hep-ph/0109214; D. H. Lyth and D. Wands, Phys. Lett. B 524, 5 (2002) arXiv:hep-ph/0110002; T. Moroi and T. Takahashi, Phys. Lett. B 522, 215 (2001) [Erratum-ibid. B 539, 303 (2002)] arXiv:hep-ph/0110096. 